\title{
Correction to: Fungal biodegradation and removal of cyanobacteria and microcystins: potential applications and research needs
}

\author{
Zakaria A. Mohamed ${ }^{1}$ (1) $\cdot$ Mohamed Hashem $^{2,3} \cdot$ Saad Alamri $^{2} \cdot$ Alexandre Campos $^{4} \cdot$ Vitor Vasconcelos $^{4,5}$ \\ Published online: 3 August 2021 \\ (C) Springer-Verlag GmbH Germany, part of Springer Nature 2021
}

Correction to: Environmental Science and Pollution Research (2021) 28:37041-37050 https://doi.org/10.1007/s11356-021-14623-w

In funding section, the correct grant number is G.R.P. $42 / 42$.

Publisher's note Springer Nature remains neutral with regard to jurisdictional claims in published maps and institutional affiliations.

A Correction to this paper has been published: https://doi.org/10.1007/ s11356-021-15673-w

The online version of the original article can be found at https://doi.org/ 10.1007/s11356-021-14623-w

Zakaria A. Mohamed

mzakaria_99@yahoo.com; zakaria.attia@science.sohag.edu.eg

1 Department of Botany, Faculty of Science, Sohag University, Sohag 82524, Egypt

2 College of Science, Department of Biology, King Khalid University, Abha 61413, Saudi Arabia

3 Faculty of Science, Botany and Microbiology Department, Assiut University, Assiut 71516, Egypt

4 CIIMAR, Interdisciplinary Centre of Marine and Environmental Research, Terminal de Cruzeiros Do Porto de Leixões, Av. General Norton de Matos, s/n, 4450-208 Matosinhos, Portugal

5 Departament of Biology, Faculty of Sciences, University of Porto, Rua Do Campo Alegre, 4169-007 Porto, Portugal 